\title{
Endoscopic Transsphenoidal Surgery for Pituitary Adenoma. Early Experience in Sohag University Hospitals
}

\author{
Karam Kenawy¹, Ahmed Ismail ${ }^{2}$, Ibrahim Rezk ${ }^{2}$, Abdin K. Kasim ${ }^{1 *}$ \\ ${ }^{1}$ Department of Neurosurgery, Sohag University, Sohag, Egypt \\ ${ }^{2}$ Department of E.N.T, Sohag University, Sohag, Egypt \\ Email: *abdin_mail@yahoo.com
}

How to cite this paper: Kenawy, K., Ismail, A., Rezk, I. and Kasim, A.K. (2021) Endoscopic Transsphenoidal Surgery for Pituitary Adenoma. Early Experience in Sohag University Hospitals. Open Journal of Modern Neurosurgery, 11, 194-203. https://doi.org/10.4236/ojmn.2021.113023

Received: June 12, 2021

Accepted: July 20, 2021

Published: July 23, 2021

Copyright $\odot 2021$ by author(s) and Scientific Research Publishing Inc. This work is licensed under the Creative Commons Attribution International License (CC BY 4.0).

http://creativecommons.org/licenses/by/4.0/

\section{(c) (i) Open Access}

\begin{abstract}
Objective: To report the results of our first series of patients undergoing a purely endoscopic endonasal approach for pituitary surgery in Sohag University Hospitals. Patients and Methods: We reviewed 20 consecutive patients during the period from January 2017 to January 2020 with pituitary adenoma who underwent purely endoscopic transsphenoidal resection of their lesions. The patients' clinical outcomes, degrees of tumor removal, and complications were reported. Results: There were 18 primary and 2 recurrent adenomas (19 nonfunctioning and 1 functioning GH secreting adenoma). The average degree of gross total removal for tumors was $70 \%$, C.S.F leak occurred in $20 \%$, diabetes insipidus in $10 \%$ and sellar hemorrhage in 5\%. There was no post-operative mortality, vascular injury, sphenoiditis, septal perforation, epistaxis or general complication. Conclusion: A purely endoscopic approach for pituitary adenoma treatment is a safe and effective alternative to the traditional microscopic procedure.
\end{abstract}

\section{Keywords}

Endoscopic Surgery, Endonasal, Pituitary Adenoma, Transsphenoidal, Outcome, Complications

\section{Introduction}

The surgical treatment of pituitary tumors through an open craniotomy was first described by Horsley [1]. Since then, pituitary surgery has undergone constant development and progress. Cushing did not use external incisions and introduce the sublabial transseptal transsphenoidal approach. In the 1960s, the era of microscopic transsphenoidal surgery started with introduction of microscope and 
fluoroscopy in surgical intervention for pituitary lesion. The transseptal sublabial approach has long been used as the standard approach because it is associated with minimal morbidity and mortality [2].

The era of endoscopic pituitary surgery was dependent on several technological advances, the most obvious of which is the endoscope. Jankowski and coworkers were first to introduce endoscopic surgery of the pituitary in 1992 [3]. But it was the landmark paper by Jho and Carrau in 1997 that marked the beginning of modern endoscopic pituitary surgery [4]. This was followed by a report by Cappabianca et al., who developed endoscopic equipment and suggested technical improvements for the approach [5].

Currently, endoscopic transsphenoidal pituitary surgery has become a preferred alternative option because of its advantages of better panoramic visualization and minimal invasiveness, which allow surgeons to gain access to different varieties of skull base lesions. One of the disadvantages of the endoscope is lacking the stereoscopic view of the microscope, and this makes the benefits of the two techniques more or less equivalent when comparing them in the treatment of pituitary adenomas.

\section{Patients and Methods}

From January 2017 to January 2020, at the Neurosurgical Department of the Sohag University Hospital, Sohag University, 20 patients underwent endonasal endoscopic transsphenoidal surgery for their pituitary adenomas. In our institution neurosurgeons operate these cases in collaboration with ENT surgeons as a team work.

\subsection{Preoperative Evaluation}

All patients underwent preoperative endocrinological, neuroradiological and neuroophthalmological evaluation.

Serum free thyroxine $\left(\mathrm{T}_{3}\right.$ and $\left.\mathrm{T}_{4}\right)$, thyrotropin (TSH), corticotropin (ACTH), prolactin (PRL), growth hormone $(\mathrm{GH})$, luteinizing hormone (LH), follicle-stimulating hormone (FSH), estradiol (in females) and serum cortisol are all studied.

To assess the size and the invasion of the adenoma, all patients underwent magnetic resonance imaging (MRI), with and without administration of intravenous contrast agent prior to surgery. CT scan of the brain and paranasal sinuses with and without contrast and CT-Angiography in large lesions were used in all patients for surgical planning. Sphenoid sinus and septal anatomy were evaluated in detail.

\subsection{Surgical Approach}

\subsubsection{Nasal Step and Sphenoidotomy}

\section{1) Patient Positioning}

Patients are positioned supine and the head is on head rest, with the head in a 
neutral position and slightly rotated to the right side for comfort of surgeons arms. The monitors used for image guidance and endoscopy are positioned directly in front of the surgeons [6].

2) Sterilization and Drapping.

Povidone iodine solution applied over the nose and upper lip then drapping of the surgical field. The nose is sterilized and then gauze soaked in adrenaline is placed in each nostril for nasal decongestion. Sterilization and drapping of the right iliac abdominal quadrant for obtaining fat, abdominal fascia and for skull base reconstruction [6].

\section{Bilateral Exposure and Creation of Nasoseptal Flap}

Bimanual dissection forms the cornerstone of microneurosurgery and is also an absolute need for endoscopic skull base surgery. To allow for bimanual dissection, a bi-nostril approach is strongly suggested for all expanded endoscopic approaches. In case of small pituitary adenoma, uni-nostril approach may be used, but we prefer to use bi-nostril approach in all our cases.

The procedure is initiated in the right naris with the removal of the middle turbinate. Then creation of nasoseptal flap according to needs on Rt side of the nose. On Lt naris, lateralization of middle turbinate is done to increase the work space [6] [7].

\subsubsection{Sphenoid Phase}

The vomer is removed from the rostrum of the sphenoid bone to create a bilateral opening in the sphenoid sinus. The middle turbinate of the left naris is generally lateralized but not resected. The natural sphenoid ostium on the left is opened and widened so that there is communication with the sphenoidotomy on the right side, thus creating wide bilateral sphenoidotomies. The lateral margins of the sphenoidotomies are extended to the level of the medial pterygoid plates. The endoscope is then returned to the right naris and a small portion $(\sim 1-2$ $\mathrm{cm}$ ) of the posterior nasal septum is resected. This represents the most critical step in the binasal approach because it facilitates bilateral instrumentation without deviation of the septum into the path of the endoscope and compromise of visualization. To optimize the available space, the endoscope is positioned superiorly at 12 o'clock, with the suction entering into the 6 o'clock position in the right naris. Dissecting instruments can be introduced into the nasal cavity through the left naris [6].

The principle of this exposure is to create a single large rectangular cavity within the sphenoid sinus. This allows for the progressive placement of the endoscope closer to the target, which is critical for visualization by allowing for the delivery of divergent light ("flash-light" effect), close to the target and its magnification. Close placement of the endoscope is also for control of bleeding [5].

The above steps are done by the ENT team.

\subsubsection{Pituitary and Sellar Phases}

Once the general exposure is achieved and bilateral sphenoidotomies are com- 
pleted, the exposure of the pituitary fossa can proceed. The sphenoidotomy is widened to include the lateral recess of the sphenoid extending lateral to the carotid canal avoiding the arteries which are defined by the CT angiography. The exposure is then extended rostrally to expose the posterior cells of the ethmoid sinus and to further define the planum-tuberculum junction. Finally, the floor of the sphenoid is reduced back to the level of the clivus. This is particularly important in the case of macroadenomas with significant suprasellar extension.

By reducing the floor of the sphenoid by drilling, a greater caudal-to-rostral trajectory into the suprasellar space is created. Any intrasphenoidal septations must then be reduced with care, because the paramedian septations often lead to the vertical canal of the ICA. The sphenoid sinus mucosa is removed and the venous bleeding is controlled by irrigation with warm saline.

This approach generates the desired single rectangular cavity and allows for the identification of key anatomical landmarks as follows: the lateral and medial optic carotid recesses (OCR), the carotid protuberance within the parasellar space, the sellar floor, tuberculum sellae, optic canals, clival recess and paraclival segment of the carotid protuberance [6].

\subsubsection{Intrasellar Dissection}

Systematic intrasellar dissection is mandatory to optimize visualization while protecting the normal gland and avoid diaphragm descent, obstructing visualization.

1) Dural Opening and Lesion Resection

The dura mater is opened using a sickle knife in the center of the sellar face (Figure 1). The opening is extended caudally and obliquely toward the 8- and 5-o'clock positions, creating the inferior flap of the opening, and rostrally towards 2- and 10-o'clock positions creating superior and inferior flap. In the case of a macroadenoma, the tumor will herniate through the inferior opening. This portion of the tumor is removed in a posterior trajectory toward the clivus-dorsum junction.

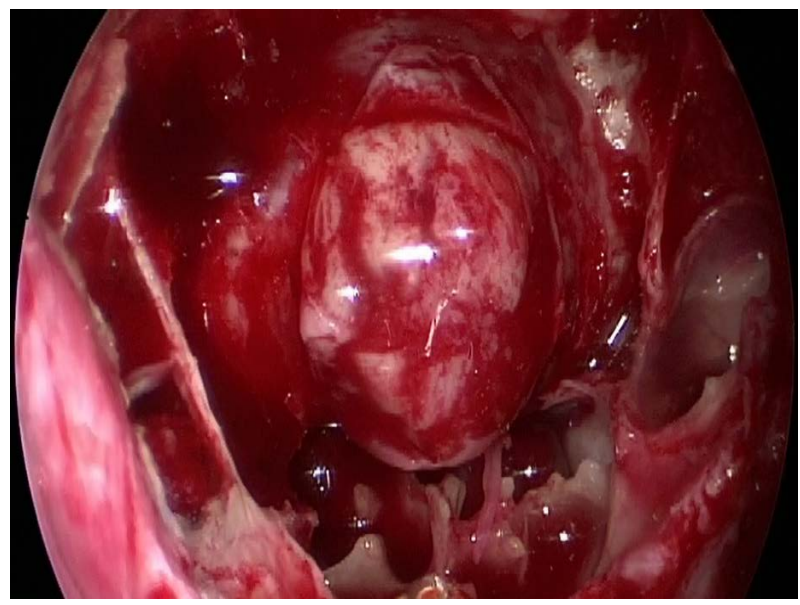

Figure 1. Intraoperative photo showing bulging of pituitary adenoma after opening of dura. 
The resection should extend widely from one cavernous sinus to the other laterally and posteriorly to the dorsum sellae. Care should be taken because the bone is defective posteriorly in many macroadenomas, and the basilar cistern can be transgressed. The pituitary stalk is most commonly located in this position.

Once the posterolateral dissection is completed, the superolateral dissection along the cavernous-carotid recess can then begin. Each recess is dissected from a caudal-to-rostral direction vertically. Before proceeding superiorly, the posterior junction of the cavernous-carotid recess should be examined and the tumor posterior to the carotid genu in the cavernous sinus can be removed. Often venous bleeding is encountered during this stage, and can be managed with packing. This should be done prior to dissecting superiorly and risking a CSF leak to avoid the exchange of blood for CSF. Once the superior lateral portion of the tumor in the sella turcica is removed, the medial OCR and angle between the optic nerve and ICA as seen from inside the sella superolaterally should be examined carefully bilaterally. This is one of the two sites most commonly found for retained tumor. The other is under the anterior lip of the dura mater at the level of the superior intercavernous sinus directly beneath the sellar/tuberculum junction.

To optimize visualization of this segment, the tumor along the anterior face overlying the diaphragma can be removed. This will allow the diaphragma to descend, opening up the superior angle between the dura mater at the level of the superior intercavernous sinus (SIS) and the anterior attachment of the diaphragma. The dura can be coagulated, shrinking it back to the SIS. These maneuvers in combination with removal of the overlying bone will optimize visualization. Final inspection of the sella turcica is undertaken in a clockwise fashion, starting inferiorly at 6 o'clock and using angled endoscopes as required. At this stage, the diaphragm should descend concentrically [6].

The most common position of the residual gland is as an apron plastered to the undersurface of the diaphragma. If the diaphragm fails to descend concentrically, this is indicative of retained tumor in the suprasellar space.

2) Cavernous Sinus Extension

Once the superior and inferior margins of the cavity have been examined, the endoscope can be placed directly within the sella to examine the medial cavernous walls that form the lateral margins of the cavity. Visualization is improved in macroadenomas by the sellar expansion but can be augmented with angled endoscopes if needed. The improved visualization facilitates the elective opening of the cavernous sinus to access tumor extension within the medial segment. The carotid siphon is usually anteriorly placed and the space between the posterior clinoid and the siphon represents an ideal entry point. Most often, the tumor creates a pathway through this space that can be followed. The tumor posterior to the ICA can be removed using the two-suction technique. As a note of caution, if there is an opening in the diaphragm (intentional or inadvertent), great 
care should be taken to avoid the exchange of blood for CSF and to avoid a subarachnoid hemorrhage with its associated complications [6].

\section{Results}

Twenty consecutive patients during the period from January 2017 to January 2020 with pituitary adenoma, underwent purely endoscopic transsphenoidal resection of their lesions.

1) Sex and Age Incidence

Out of 20 cases of different pituitary adenoma: 14 were females (70\%) and 6 were males (30\%). Age varies from 30 years to 60 years with a mean of 43.2 years.

2) Type of Lesion

There were 18 primary and 2 recurrent lesions. Of those, 19 cases had nonfunctioning and 1 functioning growth hormone secreting adenoma.

3) Clinical Symptoms and Signs

Varies from decreased vision and bitemporal hemianopia (in 19 patients; 95\%), hormonal disturbance (Acromegalic features in one patient), and headache in all patients while 2 patients presented by pituitary apoplexy as shown in Table 1.

4) Ophthalmological Results

Visual problems were seen in 19 (95\%) patients, visual improvement was seen in 17 patients $(73.6 \%)$.

5) Tumor Removal Results (Figure 2, Figure 3)

Gross total removal was achieved in 14 patients (70\%).

6) Complications

Postoperative complications are summarized in Table 2.

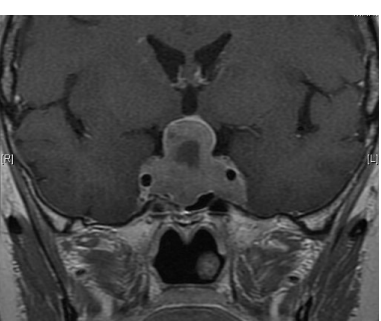

(a)

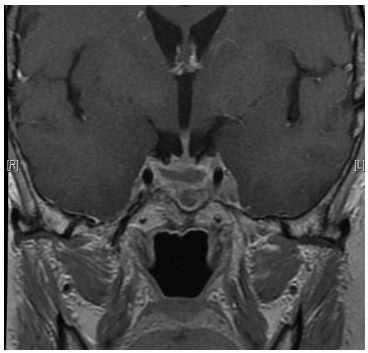

(c)

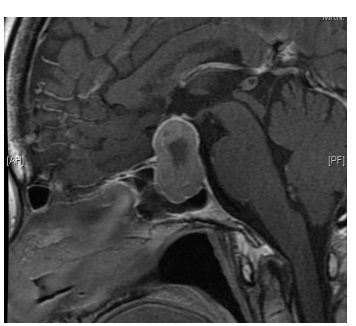

(b)

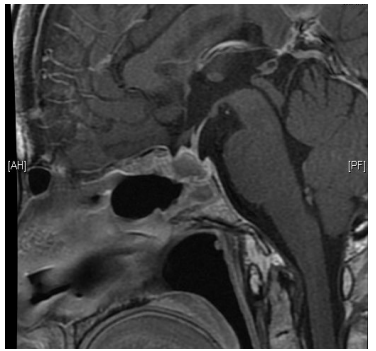

(d)

Figure 2. Case 1. With large pituitary adenoma ((a) and (b) preopeartive MRI, (c) and (d) follow up MRI 5 months post operative). 


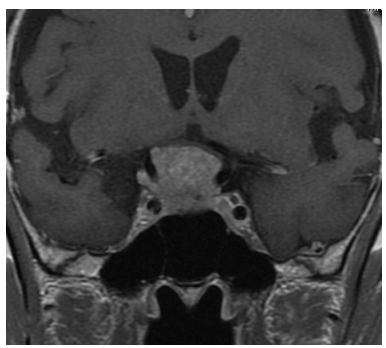

(a)

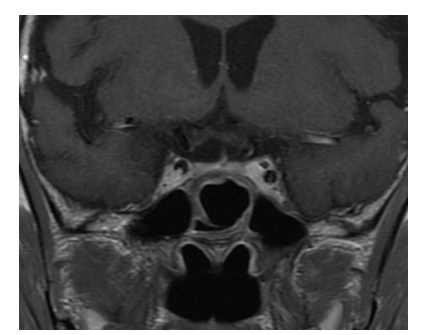

(c)

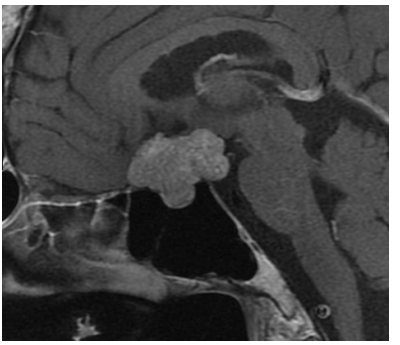

(b)

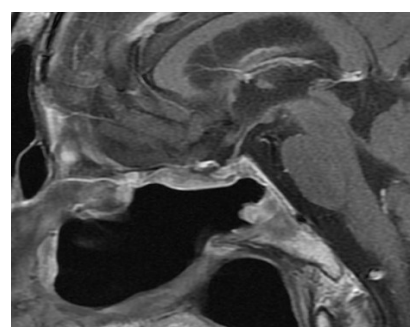

(d)

Figure 3. Case 2. With non functioning pituitary adenoma ((a) and (b) preopeartive MRI, (c) and (d) follow up MRI 3 months post operative).

Table 1. Incidence of clinical symptoms and signs.

\begin{tabular}{ccc}
\hline Clinical picture & Number & $\%$ \\
\hline Decreased visual acuity & 19 & $95 \%$ \\
Bitemporal hemianopia & 19 & $95 \%$ \\
Headache & 14 & $70 \%$ \\
Pituitary apoplexy & 2 & $10 \%$ \\
Acromegalic features & 1 & $5 \%$ \\
\hline
\end{tabular}

Table 2. Postoperative complication.

\begin{tabular}{ccc}
\hline Complications & No. of cases & $\%$ \\
\hline C. S. F leak & 4 & $20 \%$ \\
Diabetes Insipidus : & 3 & \\
1) Transient & 2 & $15 \%$ \\
2) Permanent & 1 & $5 \%$ \\
\hline Postoperative sellar HG & $10 \%$ \\
\hline
\end{tabular}

Postoperative CSF leak occurred in 4 patients (20\%). In the first 3 cases C.S.F leak was minimal and stopped spontaneously after one week of conservative treatment in the form of medical treatment (lying flat in bed, avoiding straining, cidamex and repeated lumbar puncture). In 1 case, conservative treatment failed and endoscopic reexploration was done and leak point was identified and reconstructed by abdominal fat and post operatively the patients stayed flat at bed for one week and the leak finally stopped.

Permanent Diabetes Insipidus occurred in 2 patients (10\%), and patients were on treatment by minirin tablets. Transient Diabetes insipidus occurred for about 
one week in 3 patients (15\%) and resolved spontaneously.

Post-operative sellar hemorrhage occurred in one patient (5\%) and endoscopic evacuation was done.

There was no post-operative permanent hypopituitarism, vascular injury, sphenoiditis, septal perforation, epistaxis, general complications or deaths.

\section{Discussion}

The surgery for Pituitary adenoma has developed from craniotomy toward less invasive approaches over the last century. In the last twenty years, there is a growing evidence to support the use of endoscopic approach as an alternative approach for treatment of pituitary adenomas [8] [9] [10] [11] [12]. Endoscopy can expand the surgeons' performance of transsphenoidal surgery, improves visualization and facilitates removing tumors that could not be accessed before.

Many authors have discussed the potential outcomes of the endoscopic technique. DeKlotz et al. [9], used a meta-analysis to reveal the superior rate of GTR (79\% versus $65 \%$ ) as well as the lower rates of CSF leak (5\% versus $7 \%$ ), septal perforation ( $0 \%$ versus $5 \%$ ) and post-operative epistaxis ( $1 \%$ versus $4 \%$ ), for the endoscopic approach compared with the sublabial transsphenoidal approach. Rotenberg et al. [10], concluded that the two approaches had similar outcomes (regarding GTR, hormonal abnormality resolution) but that the endoscopic approach was associated with fewer complications as well as a shorter hospital stay and length of operation. Goudakos et al. [11], demonstrated that the rates of GTR/CSF leakage were similar between the two techniques. However, the study also revealed a lower incidence of post-operative DI and a shorter hospital stay in the studied endoscopic groups. Other systematic reviews also support the safety and short-term efficacy of endoscopic pituitary surgery [8] [9] [10] [11] [12].

Gao et al. [13], favored the endoscopic approach for pituitary surgery over the microscopic approach. The endoscopic approach yielded a significantly improved rate of GTR (71.8\% for endoscopic versus 58\% for microscopic). GTR was achieved in $(83 \%)$ of patients in this series, with lower rate of post-operative septal perforation and a shorter length of hospital stay. There was no significant difference between the two approaches for meningitis, epistaxis, DI, CSF leak, hypopituitarism and the overall length of operation time. In this series GTR is $70 \%$ similar to our series.

\section{Surgical Complications}

CSF leak may occur during or after the pituitary adenoma removal. It could occur during the exploration of diaphragmatic recesses, and most of these leaks may stop with no treatment. The incidence of CSF leak increases in patients who had previous surgical interventions, or in patients who have large pituitary adenoma with suprasellar extension. The risk of CSF leak is higher in macroadenomas than microadenomas [10], due to the fact that the surgeon works closer to 
the diaphragm sellae and the subarachnoid space in these cases. So, it is important to detect the tear in the diaphragma sella and/or arachnoid membrane during the surgery and seal it [14].

In this study, any intraoperative CSF leak was repaired as soon as it was detected by abdominal fat and sealed by fibrin glue. Postoperative CSF leak occurred in 4 patients (20\%), 1 case endoscopic reexploration was done and the leak point was defined and reconstructed by fat and surgicel. In the other cases leak was minor and it stopped spontaneously within one week of conservative treatment in the form of repeated lumbar pucture, dehydrating measures, lying flat in bed and treating any causes of increased intraspinal pressure (as coughing and constipation).

Diabetes insipidus is more common following transcranial approaches than transsphenoidal surgery for giant adenomas [15]. In transsphenoidal series, the reported incidence of permanent postoperative diabetes insipidus is $8.2 \%$ to $10.4 \%$ [16]. In this series, permanent postoperative diabetes insipidus occurred in 2 patients $10 \%$, similar to the other transsphenoidal experience.

Postoperative cranial nerve dysfunction is a common complication after transcranial surgery for tumors involving the cavernous sinus, with the oculomotor nerve mostly affected [17]. However, we had no cases of permanent postoperative oculomotor or abducent nerve palsy in this series.

\section{Conclusion}

The pure endoscopic endonasal approach is a safe, effective, and minimally invasive technique for treatment of pituitary adenoma. The progression of endoscopic technology, instruments, more experienced, skilled neurosurgeons by achievement of good learning curve, and team surgery are all important factors for the good outcome of pituitary adenoma.

\section{Conflicts of Interest}

The authors declare no conflicts of interest regarding the publication of this paper.

\section{References}

[1] Horsley, V. (1887) Remarks on Ten Consecutive Cases of Operations upon the Brain and Cranial Cavity to Illustrate the Details and Safety of the Method Employed. British Medical Journal, 1, 863-865. https://doi.org/10.1136/bmj.1.1373.863

[2] Cushing, H. (1932) Intracranial Tumors: Notes upon a Series of Two-Thousand Verified Cases with Surgical-Mortality Percentages Pertaining Thereto. Charles C Thomas, Springfield, IL, 69-79.

[3] Jankowski, R., Auque, J., Simon, C., Marchal, J.C., Hepner, H. and Wayoff, M. (1992) Endoscopic Pituitary Tumor Surgery. Laryngoscope, 102, 198-202. https://doi.org/10.1288/00005537-199202000-00016

[4] Jho, H.D. and Carrau, R.L. (1997) Endoscopic Endonasal Transsphenoidal Surgery: Experience with 50 Patients. Journal of Neurosurgery, 87, 44-51. 


\section{https://doi.org/10.3171/jns.1997.87.1.0044}

[5] Cappabianca, P., Alfieri, A. and de Divitiis, E. (1998) Endoscopic Endonasal Transsphenoidal Approach to the Sella: Towards Functional Endoscopic Pituitary Surgery (FEPS). Minimally Invasive Neurosurgery, 41, 66-73. https://doi.org/10.1055/s-2008-1052019

[6] Kassam, A., Snyderman, C.H., Mintz, A., Gardner, P. and Carrau, R.L. (2005) Expanded Endonasal Approach: The Rostrocaudal Axis. Part I. Crista Galli to the Sella Turcica. Neurosurgical Focus, 19, E3. https://doi.org/10.3171/foc.2005.19.1.4

[7] Otto, B.A., Bowe, S.N., Carrau, R.L., et al. (2013) Transsphenoidal Approach with Nasoseptal Flap Pedicle Transposition: Modified Rescue Flap Technique. Laryngoscope, 123, 2976-2979. https://doi.org/10.1002/lary.24210

[8] Razak, A.A., Horridge, M., Connolly, D.J., Warren, D.J., Mirza, S., Muraleedharan, V. and Sinha, S. (2013) Comparison of Endoscopic and Microscopic Transsphenoidal Pituitary Surgery: Early Results in a Single Centre. British Journal of Neurosurgery, 27, 40-43. https://doi.org/10.3109/02688697.2012.703353

[9] DeKlotz, T.R., Chia, S.H., Lu, W., Makambi, K.H., Aulisi, E. and Deeb, Z. (2012) Meta-Analysis of Endoscopic versus Sublabial Pituitary Surgery. Laryngoscope, 122, 511-518. https://doi.org/10.1002/lary.22479

[10] Rotenberg, B., Tam, S., Ryu, W.H. and Duggal, N. (2010) Microscopic versus Endoscopic Pituitary Surgery: A Systematic Review. Laryngoscope, 120, 1292-1297. https://doi.org/10.1002/lary.20949

[11] Goudakos, J.K., Markou, K.D. and Georgalas, C. (2011) Endoscopic versus Microscopic Transsphenoidal Pituitary Surgery: A Systematic Review and Meta-Analysis. Clinical Otolaryngology, 36, 212-220. https://doi.org/10.1111/j.1749-4486.2011.02331.x

[12] Tabaee, A., Anand, V.K., Barron, Y., Hiltzik, D.H., Brown, S.M., Kacker, A., Mazumdar, M. and Schwartz, T.H. (2009) Endoscopic Pituitary Surgery: A Systematic Review and Meta-Analysis. Journal of Neurosurgery, 111, 545-554. https://doi.org/10.3171/2007.12.17635

[13] Gao, Y., Zhong, C., Wang, Y., Xu, S., Guo, Y., Dai, C., et al. (2014) Endoscopic Versus Microscopic Transsphenoidal Pituitary Adenoma Surgery: A Meta-Analysis. World Journal of Surgical Oncology, 12, 94. https://doi.org/10.1186/1477-7819-12-94

[14] Berker, M., Hazer, D.B., Yucel, T., Gurlek, A., Cila, A., Aldur, M. and Onerci, M. (2012) Complications of Endoscopic Surgery of the Pituitary Adenomas: Analysis of 570 Patients and Review of the Literature. Pituitary, 15, 288-300. https://doi.org/10.1007/s11102-011-0368-2

[15] Buchfelder, M. and Kreutzer, J. (2008) Transcranial Surgery for Pituitary Adenomas. Pituitary, 11, 375-384. https://doi.org/10.1007/s11102-008-0120-8

[16] de Paiva Neto, M.A., Vandergrift, A., Fatemi, N., Gorgulho, A.A., Desalles, A.A., Cohan, P., et al. (2010) Endonasal Transsphenoidal Surgery and Multimodality Treatment for Giant Pituitary Adenomas. Clinical Endocrinology (Oxford), 72, 512-519. https://doi.org/10.1111/j.1365-2265.2009.03665.x

[17] Dolenc, V.V. (1997) Transcranial Epidural Approach to Pituitary Tumors Extending beyond the Sella. Neurosurgery, 41, 542-552.

https://doi.org/10.1227/00006123-199709000-00007 